\title{
Locally Pressed Fibre Bragg Grating Pressure Sensor
}

\author{
Ricardo Correia, Edmon Chehura, Stephen W. James and Ralph P. Tatam \\ Optical Sensors Group, School of Engineering, Cranfield University, Bedford MK43 OAL, UK \\ Author e-mail address: s.w.james@cranfield.ac.uk
}

\begin{abstract}
A spectral-hole created by locally pressing a fibre Bragg grating (FBG)s exhibited a wavelength-shift within the FBG bandwidth. A sensitivity of $74.3 \mathrm{pm} /(\mathrm{N} / \mathrm{mm})$ was obtained for pressure applied along the slow axis of a high birefringence fibre. (C)2006 Optical Society of America

OCIS codes: (060.2370) Fiber optics sensors; (060.2420) Fibers, polarization-maintaining
\end{abstract}

\section{Introduction}

Transversely loaded fibre Bragg gratings (FBGs) have been proposed for use as multi-axis strain sensors and for the monitoring of the cure of composite materials [1]. When a transverse load is applied uniformly along the axis of an FBG fabricated in single mode (SM) optical fibre, the introduction of birefringence causes a splitting of the Bragg peak. In the case of FBGs fabricated in highly birefringent (HiBi) optical fibre the separation of the central wavelengths of the two orthogonally polarised Bragg peaks increases or decreases, depending upon the orientation of the load with respect to the birefringent axes of the fibre. The response of FBGs fabricated in a range of (HiBi) optical fibre types to transverse loading has been the subject of a detailed study [1-3]. In general the highest sensitivity is obtained when the load is applied along the eigen-axes of the HiBi fibre.

Non-uniform loading of FBGs has been shown to introduce often unwanted features into the Bragg spectrum. However, this can be used to manipulate and optimise the spectrum for particular applications. Non-uniformly transverse loading of an FBG introduces a spectral hole within the bandwidth of the FBG [4-6]. This paper analyses this effect with the aim of developing a pressure sensor.

\section{Sensing Principle.}

The principle of operation of the pressure sensor is based upon transducing the pressure into a local loading of the central section of an FBG via a piston. The loading modifies the local refractive index and dimensions of the fibre and thus the Bragg condition. The FBG has three distinct regions, with the unperturbed regions forming a cavity. This configuration has been analysed using both the transfer matrix and Rouard's methods [7]. A typical Bragg spectrum, for a load of $1.33 \mathrm{~N} / \mathrm{mm}$ applied along a $1.5 \mathrm{~mm}$ length of a $5 \mathrm{~mm}$ long FBG is shown in figure 1 , illustrating the creation of a hole within the Bragg spectrum. The wavelength of the spectral hole is dependent upon the applied load, and shows a cyclical response.

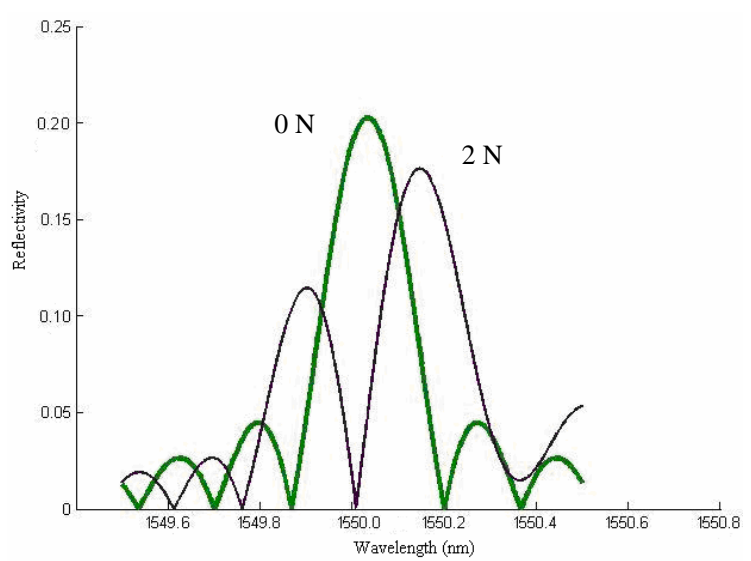

Figure 1: Spectrum of an FBG, of total length $5 \mathrm{~mm}$, subject to a transverse load applied along a $1.5 \mathrm{~mm}$ long section at its centre. 


\section{Experiment}

FBG's were fabricated in photosensitive single mode (SM) optical fibre (fibrecore PS1250) and in a highly birefringent bow-tie optical fibre (Fibercore HB1500). The FBGs were of length $6 \mathrm{~mm}$ and 3mm respectively and had centre wavelengths of 1550nm. The experimental arrangement used to interrogate the transverse loaded FBG is shown schematically in Figure 4. The wavelength range of interest was scanned with a resolution of $3 \mathrm{pm}$ using a tunable external cavity laser (TUNICS-Plus CL) coupled to a Polarisation Maintaining (PM) coupler. The FBG was attached to one output arm of the PM coupler while the other output was immersed in an index matching gel. The light reflected from the FBG was directed to a PM fibre splitter. The two orthogonal linear polarisation outputs of the splitter are recorded separately by two photo-detectors via a data acquisition (DAQ) card. All optical components in the interrogation system were connected using standard telecommunications FC/PC connectors that were index matched.

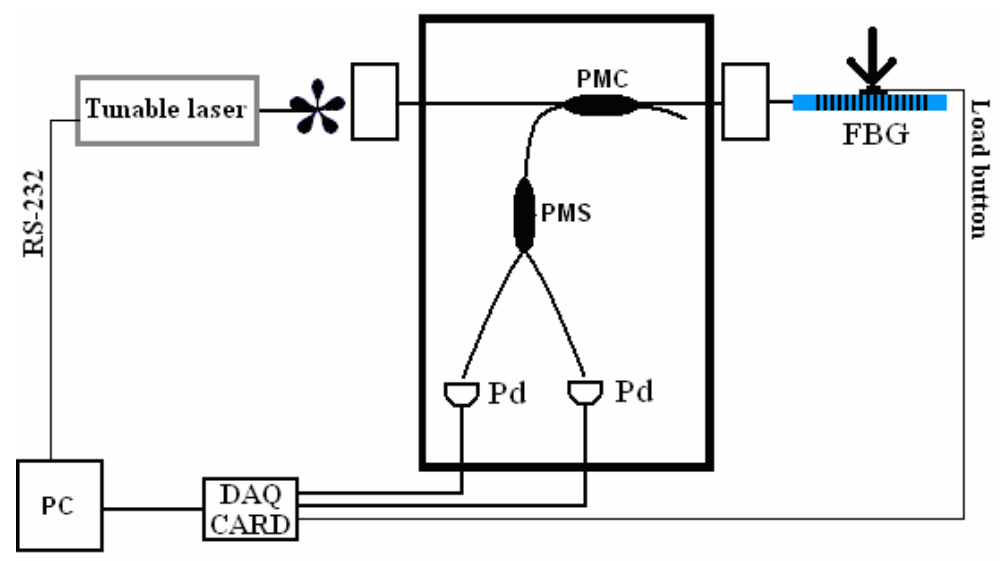

Figure 2. Schematic diagram of the experimental arrangement

In order to apply the pressure to the fibre, a loading fixture designed to minimise the fibre twist (as represented in Figure 3), used previously to characterise the transverse load response of PM FBGs [1], was employed. The above mentioned test fibre and an identical support fibre located parallel to the first are loaded transversally between the two metal stages that were designed to apply a load to $1.05 \mathrm{~mm}$ of the FBG. This stage has three contact points, one located at the centre of the FBG, with a length of $1.05 \mathrm{~mm}$ and two other contact points, each of length $5 \mathrm{~mm}$, which are outside the FBG. The load was applied to the fibres by rotating a screw, and monitored by a load button with a capacity of $222 \mathrm{~N}$ and resolution of $0.1 \mathrm{~N}$. The signal from the load button was recorded through a DAQ card. The alignment of the fibre polarisation axes within the loading fixture was positioned through two rotational stages.

The centre of the FBG was approximately determined during the fabrication process and later confirmed applying a fixed load at different positions along the FBG and analysing the reflectivity spectrum. When the load was applied at the centre of the FBG, the spectral hole showed the largest sensitivity.

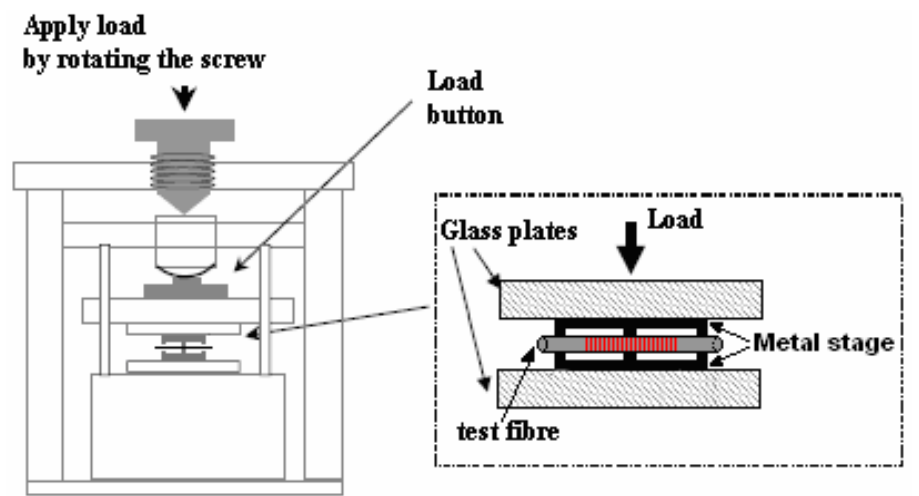

Figure 3. Representation of the loading fixture 


\section{Results and discussion}

Figure 4 shows the response of the spectrum of the SM FBG to the application of the transverse load. The spectral hole tracks across the spectrum, as predicted theoretically, with a sensitivity of $50 \mathrm{pm} / \mathrm{N} / \mathrm{mm}$. There is evidence of the introduction of birefringence by the growth of the spectral feature at the red end of the spectrum. To investigate the birefringence effects, a HiBi FBG was subjected to the transverse load, aligned with the slow axis of the fibre. The reflection spectra obtained from the slow eigen-axis shown in Figure 5. It was observed that the spectral hole appeared only along the loaded axis i.e. in this case, the slow axis of the FBG spectrum. The spectrum obtained from the fast axis was unperturbed. The spectrum hole exhibited a red shift with increasing load. Once the spectral hole had traversed the FBG bandwidth, a new cycle started, with the spectral hole reappearing at the blue end of the spectrum.

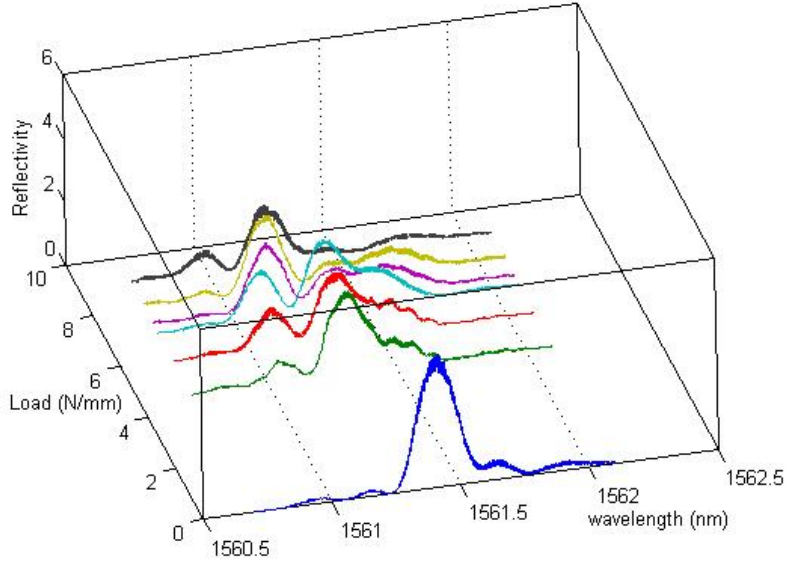

(a)

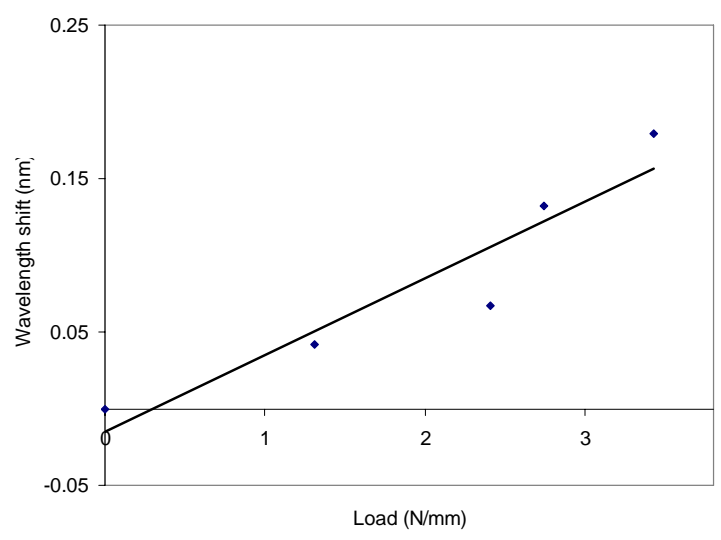

(b)

Figure 4 (a) Evolution of the reflection spectrum from a SM FBG subject to a local transverse load applied along a 1.05mm length, at the centre of theFBG . (b) Dependence of the central wavelength of the spectral hole on applied load

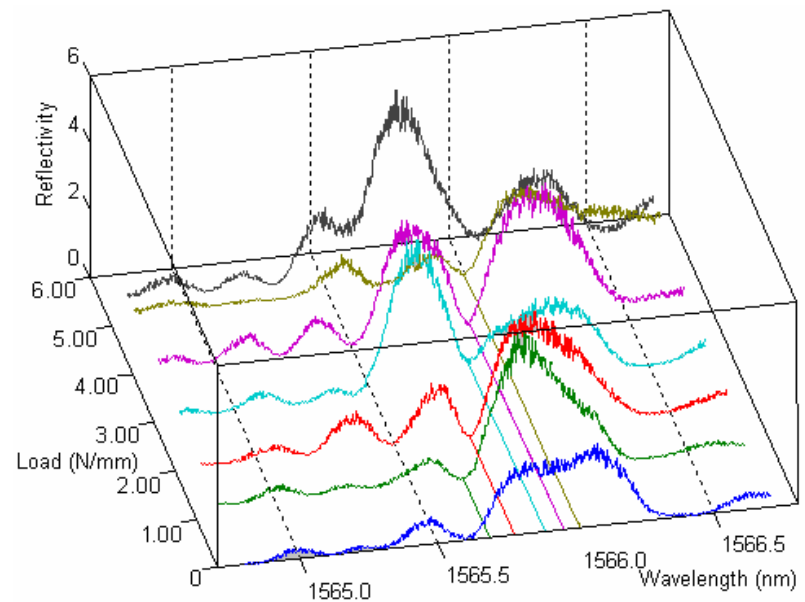

(a)

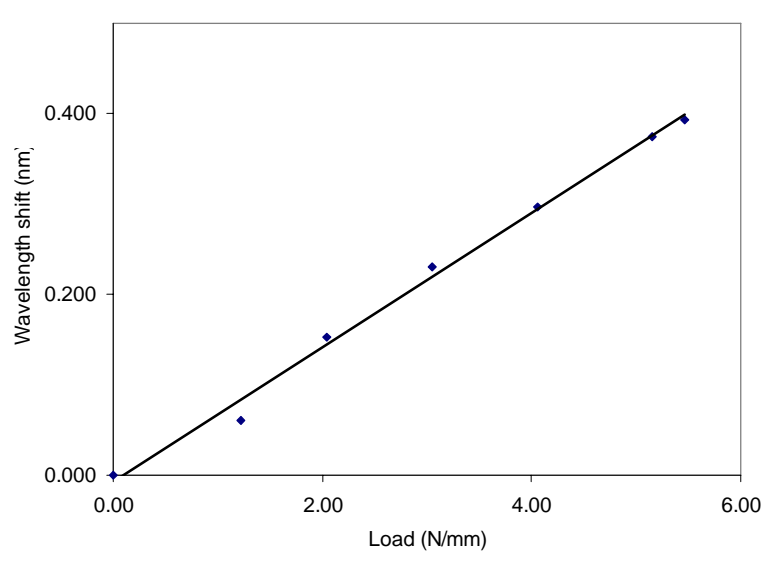

(b)

Figure 5 (a) Evolution of the reflection spectrum from the slow axis of the HiBi FBG subject to a local transverse load applied along a 1.05mm length, aligned with the slow axis. (b) Dependence of the central wavelength of the spectral hole on applied load.

The dependence of the central wavelength of the spectral hole within the FBG bandwidth upon the applied load is illustrated in Figure 5, demonstrating a sensitivity of $74.3 \mathrm{pm} /(\mathrm{N} / \mathrm{mm})$. The total length of fibre in contact with the metal stage was $11.01 \mathrm{~mm}$. Thus the load imposed to the $1.05 \mathrm{~mm}$ length of the FBG was only approximately 1/11 of the total load. The sensitivity could be enhanced by decreasing the length of the contact points. 


\section{Conclusion}

Local transverse loading of FBGs has been observed to introduce a spectral hole into the Bragg spectrum. This effect has been analysed for FBGs fabricated in SM and HiBi optical fibres. The spectral hole developed in the SM fibres showed a sensitivity of 50pm/ $/ \mathrm{mm}$, and demonstrated the cyclical behaviour predicted by a theoretical analysis of the system. An investigation of locally loaded HiBi FBGs showed that the spectral hole appears only in the Bragg reflection from the axis along which the load is applied.

\section{Acknowledgements}

The authors gratefully acknowledge the support of the Royal Society Paul Instrument Fund, UK

\section{References}

[1] Chehura, E, Chen-Chun Y., Staines, S. E., James, S. W., and Tatam, R. P. Characterization of the response of fibre Bragg gratings fabricated in stress and geometrically induced high birefringence fibres to temperature and transverse load. Smart Materials and Structures 13, 888-895. 2004.

[2] Bossia, Frederico, Giaccari, Philippe, Botsis, John, Facchini, Mauro, Limberger, Hans, and Salathé, René. Characterization of the response of fibre Bragg grating sensors subjected to a two-dimensional strain field. Smart materials and Structures 12, 925934. 2003.

[3] Lawrence, C., nelson, D., and Udd, E. Measurement of transverse strains with fiber Bragg gratings. SPIE 3042, 218-228. 97. [4] Matos, C. J. S, Torres, P., Valente, L. C. G., Margulis, W., and Stubbe, R. Fiber Bragg Grating (FBG) Characterization and Shaping by Local Pressure. Journal of Lightwave Technology 19(8), 1206-1211. 2001.

[5] Michaille, L., mccall, M. W., Lai, Y. C., and Williams, J. A. R. Analysis of single and multiple, non-permanent, tunable, birefringent spectral holes in a fibre-Bragg grating stop-band produced via uniaxial pressure. Optics Communications 222, 1-8. 2003.

[6] Torres, P. and Valente, L. C. G. Spectral response of locally pressed fiber Bragg grating. Optics Communications , 285-291. 2002.

[7] Chehura, E., James, S. W., and Tatam, R. P. Rouard's method as a modelling tool for the sensing characteristics of complex fibre Fabry-Perot interferometers formed between chirped fibre Bragg Gratings. In press, Journal of Lightwave Technology 